\title{
Impact of Beta Blockers and HCN Blockers on C - reactive Protein in CAD Patients
}

\author{
M.Ranga Priya*, Ayshwariya Laxmi, Ilakya.G, Irene George, Irine Jacob \\ Department of Pharmacy Practice, Swamy Vivekanandha College of Pharmacy, Tiruchengode, Tamil Nadu, India. \\ *Corresponding author's E-mail: priyanarayan97@gmail.com
}

Received: 05-06-2020; Revised: 17-08-2020; Accepted: 28-09-2020; Published on: 20-10-2020.

\begin{abstract}
The elevated levels of various inflammatory markers in CVD are having increased recognition in the recent days. Among all inflammatory markers, CRP has been most deeply interrogated in clinical studies. CRP is an inflammatory marker which is an independent predictor of adverse cardiac events including death, need for transplantation etc. CRP levels have been identified to predict future risk in patients with CAD. A Prospective Observational Study was conducted for 6 months period to assess the variation in C-reactive protein level in CAD patients under Beta blocker and HCN channel blocker therapy and to check the effectiveness of the drug in controlling the C-reactive protein levels. CRP level was analyzed using enzyme immuno assay among inpatients admitted with CVD. The demographic details and treatment data of the 150 patients were collected in a specially designed proforma. The age of study population was found in between 50-80years. The prevalence of CAD was higher in males than females. In CAD patients with Beta blocker and HCN blocker treatment, the CRP level was decreased comparatively in patients who were under Beta blockers therapy than in patients using $\mathrm{HCN}$ channel blockers. The present study concluded that Beta blocker therapy has increasingly suppressed circulating CRP concentrations when compared with HCN channel blockers in patients who exhibited higher baseline CRP concentrations.
\end{abstract}

Keywords: Coronary Artery Disease, C-reactive protein, Cardio vascular Disease, Beta blockers, HCN channel blockers.

QUICK RESPONSE CODE $\rightarrow$
DOI:
10.47583/ijpsrr.2020.v64i02.006
DOI link: http://dx.doi.org/10.47583/ijpsrr.2020.v64i02.006

\section{INTRODUCTION}

C $A D$, also known as coronary heart disease, is the narrowing of the blood vessels that supply blood and oxygen to the heart, as a result of atherosclerosis which can lead to unstable angina, myocardial infarction (MI), and heart failure. CAD encloses stroke peripheral artery disease, aortoiliac disease and carotid artery disease. CAD is a leading cause of mortality worldwide, a serious economic burden on healthcare systems. Inflammation plays a role in pathogenesis of onset and progression of atherosclerosis. ${ }^{1}$ Cardiovascular diseases led to nearly 17.5 million deaths in the year 2012 globally. According to a WHO report in 2014, the ageadjusted cardiovascular disease mortality rates in India were 349 and 265 per 100000 in men and women. These rates are more than two to three times higher than in USA (170 and 108 per 100000 in men and women, respectively). ${ }^{2}$ Inflammation is considered to be a crucial factor of the pathophysiology of coronary heart disease (CHD), and the inflammatory cascade is particularly important in the atherosclerotic process. Atherosclerosis characterised by a complex multifactorial pathophysiology. Inflammation in the vessel wall plays a major role in initiation, progression and in the end steps of atherosclerosis called as plaque destabilisation and plaque rupture which is considered as an inflammatory response to injury and endothelial dysfunction. Any damage to the endothelial wall triggers a cascade of events that alters the inflammatory response leading to the enrolment of white blood cells into the blood vessel wall which give rise to abnormal foam cells and begin the development of atherosclerotic lesions. ${ }^{3}$ Serum levels of high-sensitivity Creactive protein (hs-CRP), interleukin-6 (IL-6), and tumor necrosis factor- $\alpha$ (TNF- $\alpha$ ) have been shown to be predictors of adverse result in patients with coronary artery disease (CAD). We hypothesized that measurement of inflammatory markers could predict atherosclerotic burden and major cardiac events. ${ }^{4}$ Inflammatory markers may have greater potential in the description of individuals who should be treated with lipid lowering, antiplatelet or any other cardio vascular drug therapy to assess the risk. Monitoring the effects of therapy such as HMG CoA reductase inhibitor might be considered as one of the potential use of inflammatory markers. For example treatment might be exacerbated if the marker does not fall. ${ }^{5} \mathrm{C}$ protein was the early prognosis of the body's chemical response to inflammatory mediators and result in "acute phase protein". The elevated levels of various inflammatory markers in CVD are having increased recognition. Among all inflammatory markers, CRP has been most deeply interrogated in clinical studies. CRP levels has been identified to predict future risk in patients with CAD. ${ }^{6}$ CRP called as classic acute phase protein which is not directly taken place in the coagulation process still is considered to be a sensitive marker of tissue damage and

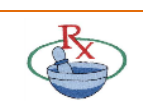


inflammation. Serum concentration of cytokine II 6 is a major cytokine of the acute phase response which are considered as predictive of an initial cardiovascular event. CAD patients are identified from high levels of TNF at risk for recurrent cardiovascular activity. Cytokines may be more proximate to the underlying inflammatory process than fibrinogen or CRP. In stable patients with CAD, cytokines levels may be undetectable in plasma samples ${ }^{7}$

Elevated levels of CRP-is associated with:

- Underlying atherosclerosis

(subclinical atherosclerosis)

- Increased risk of a first cardiovascular event among individuals with or without risk factors

- Increased risk of recurrent cardiovascular events

- Diabetes

- Obesity

- Metabolic syndrome

- New-onset hypertension

- Sedentary lifestyle

- Heart failure

CRP is a risk marker and not a histological risk factor for CHD which will provide prognostic information independent of lipid parameters in healthy individuals. ${ }^{8}$

\section{MATERIALS AND METHODS}

The study was a Prospective Observational Study conducted at Vivekanandha Medical Care Hospital, Elayampalayam, Tiruchengode with the approval of the Institutional Ethical Committee (Ref.No.: SVCP/IEC/JAN/2019/02 dated 03/01/2019) after obtaining prior consent from the study participants for a period of 6 months (January 2019- June 2019).

\section{Study Population}

About 150 cardiac patients were screened and 90 patients were included in this study after getting their consent. Patients of age 50 and above, CAD patients who are under Beta blocker therapy, CAD patients who are under HCN channel blockers medication and patients of both genders were included in the study. Information such as demographic details, past medical and medication history was collected by chart review. The patients were divided into 3groups. Group A - Beta Blockers (30 patients); Group B - HCN Channel Blockers (30 Patients); Group C - Control Group (30 patients)

\section{RESULTS}

The study was conducted with 90 CAD patients in the department of cardiology. The patients were divided into 3 groups.

Group A - Beta Blockers (30 patients)

Group B - HCN Channel Blockers (30 Patients)
Group C - Control Group (30 patients)

\section{Age wise distribution of CAD patient}

Age distribution of the patients shows 37(41.1\%) were in the age group of $50-59$ years, $35(38.8 \%)$ were in the age group of $60-69$ and 18 (20\%) were in the age group of 70 79. Age distribution shows that patients $>50$ are prone to have CAD compared to other two groups.

Table 1: Age Wise Distribution

\begin{tabular}{|l|c|c|c|}
\hline \multicolumn{1}{|c|}{ Groups } & \multicolumn{3}{|c|}{ No. of patients (n=90) } \\
\hline Age & $50-59$ & $60-69$ & $70-79$ \\
\hline Beta blockers & 13 & 6 & 11 \\
\hline HCN blockers & 11 & 12 & 7 \\
\hline Control & 13 & 17 & 0 \\
\hline Percentage (\%) & 41.1 & 38.8 & 20 \\
\hline
\end{tabular}

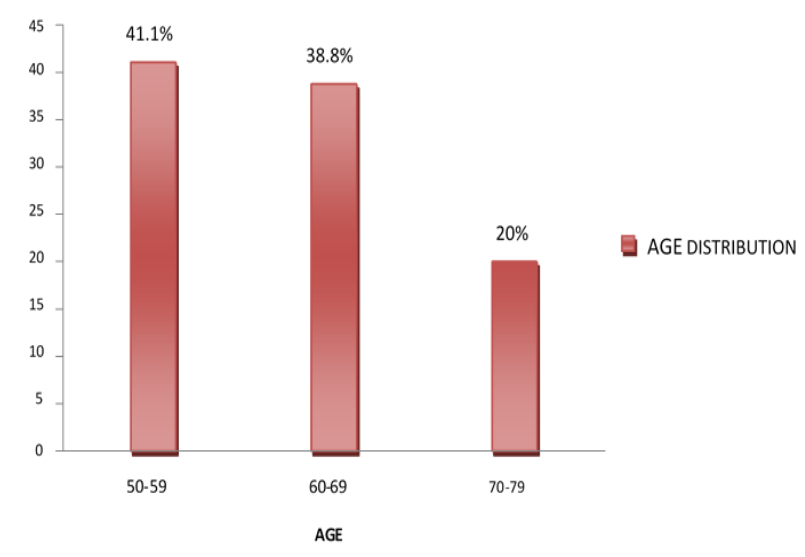

Figure 1: Age Wise Distribution

CRP range according to the betablocker and $\mathrm{HCN}$ channel blockers therapy and control group

Out of 90 patients 30 were under Beta blocker therapy (Group A), 30 were under Ivabradine therapy (Group B) and 30 were control group (Group C). The mean of all the three groups have been decreased from base line. The mean of beta blocker therapy at baseline was $14.86 \pm 2.28$ and after 20 days it was $10.89 \pm 1.9$. The mean of Ivabradine therapy at baseline was $15.14 \pm 1.51$ and after 20 days it was $12.0 \pm$ 1.0. The mean of control group at baseline was $12.68 \pm 1.92$ and after 20 days it was $11.26 \pm 2.0$. So, it is known that beta blocker therapy decreased CRP level more compared to other two groups.

Table 2: Mean Value of CRP Levels in CAD Patients on Treatment

\begin{tabular}{|c|c|c|}
\hline \multirow{2}{*}{ Therapy } & \multicolumn{2}{|c|}{ Mean CRP Level } \\
\hline & Baseline & After 20 days \\
\hline Betablockers & $14.86 \pm 2.28$ & $9.9 \pm 1.97$ \\
\hline Ivabradine & $15.14 \pm 1.51$ & $12.0 \pm 1.00$ \\
\hline Control & $12.68 \pm 1.92$ & $11.8 \pm 2.04$ \\
\hline
\end{tabular}




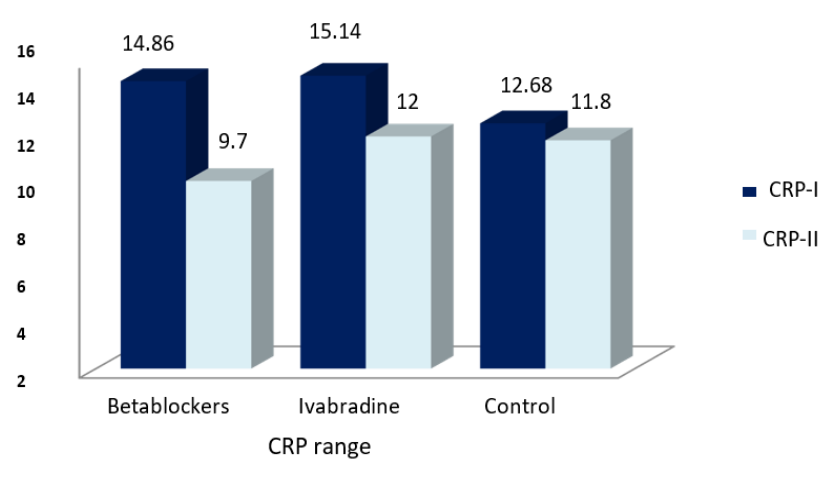

Figure 2: Mean Value of CRP Levels in CAD Patients On Treatment

\section{Comparison of ivabradine therapy and betablocker therapy}

Among 90 patients, 30 patients belong to Ivabradine therapy and 30 patients belong to Beta blocker therapy. While comparing the CRP level in both the groups, it is found that Beta blocker therapy showed much more decrease in CRP level than Ivabradine group.

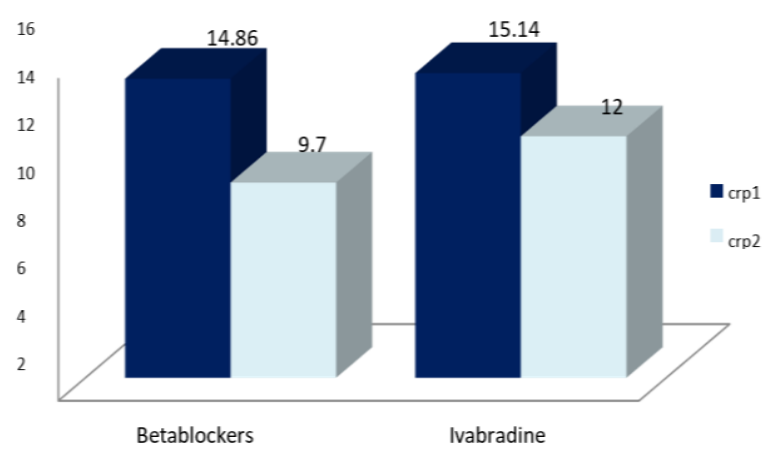

Figure 3: Mean Value of CRP In Beta Blocker and Ivabradine

\section{DISCUSSION}

CAD is the narrowing of the blood vessels, as a result of atherosclerosis that supply blood and oxygen to the heart that can lead to unstable angina, myocardial infarction (MI) and heart failure. CAD encloses stroke peripheral artery disease, aortoiliac disease and carotid artery disease. CAD is a leading cause of mortality worldwide, a serious economic burden on healthcare systems ${ }^{1}$. Inflammation plays a role in pathogenesis of onset and progression of atherosclerosis. C-reactive protein (CRP) is a non-specific biological marker of inflammation and used in the detection of patients at increased risk for cardiovascular disease ${ }^{9}$. CRP is a risk marker for CHD which will provide prognostic information independent of lipid parameters in healthy individuals ${ }^{8}$. CAD is higher in males (69\%) than females (31\%). In our study, it depicts that the occurrence of CAD is highest in patients within the age category of 50-59 i.e. 37 patients (41.1\%).

The CRP level was recorded in 90 patients, among whom, 30 patients were under Beta blocker therapy (group A), 30 patients were under Ivabradine therapy (group B) and 30 were control group (group C). The mean of all the three groups have been decreased from base line. The mean of beta blocker therapy at baseline was $14.86 \pm 2.28$ and after 20 days it was $10.89 \pm 1.9$. The mean of Ivabradine therapy at baseline was $15.14 \pm 1.51$ and after 20 days it was $12.0 \pm$ 1.0. The mean of control group at baseline was $12.68 \pm 1.92$ and after 20 days it was $11.26 \pm 2.0$. Thus, it is evident that betablocker therapy decreased CRP level more when compared to the other two groups. This study is in agreement with the study conducted by Nagatomo $Y$ (2007) suggesting that the CRP concentration decreased significantly in patients with higher baseline CRP concentration, under Carvedilol therapy ${ }^{10}$.

This is in accordance with the study done by Nicholas $P$. Jenkins (2002) which concluded that patients prescribed beta-blockers had significantly lower mean C-reactive protein concentrations than in patients to whom those were not prescribed. So, Beta-blockers may affect Creactive protein concentrations in CAD patients ${ }^{11}$.

The study is associated with the study done by Yusuke Jo (2008). The study suggests that early use of beta- blockers prevented excessive inflammation after distal type acute aortic dissection (AAD), suggesting a pleiotropic effect of beta-blockers on the inflammatory response after $A A D^{12}$.

A similar study was conducted by Mary S Beattie (2009) which suggest $\beta$-blockers have been shown to reduce inflammation through decreases in sympathetic tone. CRP is a marker of an inflammatory process that causes ischemia, use of 6 -blockers may prevent this inflammatory process from promoting atherogenesis and ischemia. Inflammation and CRP are part of a reaction to ischemia, b-blockers may decrease this inflammatory response by reducing the severity of ischemia ${ }^{13}$.

\section{CONCLUSION}

CRP is an inflammatory marker which is an independent predictor of adverse cardiac events including death, need for transplantation etc. The present study concludes that Beta blocker therapy has increasingly suppressed circulating CRP concentrations when compared with HCN channel blocker in patients who exhibit higher baseline CRP concentrations.

\section{REFERENCES}

1. Shrivastava AK, Singh HV, Raizada A, Singh SK, C-reactive protein, inflammation and coronary heart disease, The Egyptian Heart Journal, 67, 2015,1-9.

2. Gupta R, Mohan I, Narula J, Trend in Coronary Heart Disease Epidemiology in India., Annals of Global Health, 82, 2016, 307-315.

3. Singh HV, Raizada A, Singh SK, C-reactive protein, inflammation and coronary heart disease, The Egyptian Heart Journal, 67, 2015, 1-9.

4. Sukhija R, Fahdi I, Garza L, Fink L, Inflammatory Markers, Angiographic Severity of Coronary Artery Disease, and 
Patient Outcome, The American Journal of Cardiology, 99, 2007, 879-884.

5. Pearson T A, Mensah G A, Wayne Alexander. R, Markers of Inflammation and Cardiovascular Disease, Journal of American Heart Association, 107, 2003, 499-511.

6. Jun Lia J, Hong Fanga C, C-reactive protein is not only anti- inflammatory marker but also a direct cause of cardiovascular diseases, Journal of Medical Hypothesis, 62, 2004, 499-506.

7. Rosenson R S, Koenig W, Utility of Inflammatory Markers in the Management of Coronary Artery Disease, American journal of cardiology, 92, 2003, 10-18.

8. Krintus M, Value of C-Reactive Protein as a Risk Factor for Acute Coronary Syndrome: A Comparison with Apo lipoprotein Concentrations and Lipid Profile, Mediators of Inflammation, 2012, 1-10.

9. Clearfield MB, C-Reactive Protein: A New Risk Assessment Tool for Cardiovascular Disease, The Journal of the American Osteopathic Association, 105, 2005, 409416.

10. Lagerqvist $B$, Diderholm $E$, Lindahl B, Husted S, Kontny $F$, Stahle E, Swahn E,Venge $P$, Siegbahn A, Wallentin L,FRISC score for selection of patients for an early invasive treatment strategy in unstable coronary artery disease, Heart Journal, 91, 2005, 1047-1052.

11. Jenkins N P, Keevil BG, Hutchinson I V, Beta-blockers Are Associated with Lower C-Reactive Protein Concentrations in Patients with Coronary Artery Disease, The American Journal of Medicine, 112, 2002, 269-274.

12. Jo $Y$, Anzai T, Sugano $Y$, Naito $K$, Ueno K, Kohno T, Yoshikawa T, Ogawa S, Early use of beta-blockers attenuates systemic inflammatory response and lung oxygenation impairment after distal type acute aortic dissection, Heart Vessels, 23, 2008, 334-340.

13. Beattie M S, Shlipak M G, Liu H, Browner W S, Nelson $B, C$-Reactive Protein and Ischemia in Users and Nonusers of $\beta$-Blockers and Statins: Data from the Heart and Soul Study, National Institute of Health, 107, 2003, 245-250.

Source of Support: None declared.

Conflict of Interest: None declared.

For any question relates to this article, please reach us at: editor@globalresearchonline.net New manuscripts for publication can be submitted at: submit@globalresearchonline.net and submit_ijpsrr@rediffmail.com 